\section{Material Properties ANalysis of STRUCTURAL MEMBERS IN PUMPKIN BALLOONS}

\author{
W.J. Sterling ${ }^{*}$ \\ NASA Goddard Space Flight Center, \\ Wallops Flight Facility, Wallops Island, VA 23337
}

\section{ABSTRACT}

The efficient design, service-life qualification, and reliability predictions for lightweight aerospace structures require careful mechanical properties analysis of candidate structural materials. The demand for highquality laboratory data is particularly acute when the candidate material or the structural design has little history. The pumpkin-shaped super-pressure balloon presents both challenges. Its design utilizes load members (tendons) extending from apex to base around the gas envelope to achieve a lightweight structure. The candidate tendon material is highly weight-efficient braided HM cord. Previous mechanical properties studies of $\mathrm{Zylon}^{\circledR}$ have focused on fiber and yarn, and industrial use of the material in tensile applications is limited. For high-performance polymers, a carefully planned and executed properties analysis scheme is required to ensure the data are relevant to the desired application. Because no directly-applicable testing standard was available, a protocol was developed based on guidelines from professional and industry organizations. Due to the liquid-crystalline nature of the polymer, the cord is very stiff, creeps very little, and does not yield. Therefore, the key material property for this application is the breaking strength. The pretension load and gauge length were found to have negligible effect on the measured breaking strength over the ranges investigated. Strain rate was found to have no effect on breaking strength, within the range of rates suggested by the standards organizations. However, at the lower rate more similar to ULDB operations, the strength was reduced. The breaking strength increased when the experiment temperature was decreased from ambient to $183 \mathrm{~K}$, which is the lowest temperature ULDB is expected to experience. The measured strength under all test conditions was well below that resulting from direct scale-up of fiber strength based on the manufacturer's data. This expected result is due to the effects of the braiding process and material ageing.

Keywords: ULDB, PBO cord, rope tensile strength, stratospheric balloon

\footnotetext{
* Materials Engineer, Balloon Research \& Development Laboratory; Jerry.Sterling@nasa.gov. Member.
}

\section{INTRODUCTION}

The ultra-long duration balloon (ULDB) project was initiated by the NASA Balloon Program Office to address calls for longer duration flights with less altitude variation. Present scientific balloons are of open design in that the gas pressure inside the envelope is the same as the outside atmospheric pressure. Pressure imbalance, such as might be created by solar heating during the daytime, is avoided by open vents. However, gas so purged during the day implies gradually lowering altitude over diurnal cycles. To help maintain altitude, these 'zero-pressure balloons' carry ballast that is dropped as needed. Altitude fluctuation and flight duration are thus linked by the constraint of finite ballast capacity.

The ULDB design concept being pursued by BPO is a pressurized (superpressure) balloon with a closed envelope and nominally constant volume. Daytime heating is thus accommodated by internal pressure increase. In the ideal case where the balloon shape is unaffected by such pressure changes, the balloon volume, buoyancy, and altitude are steady. ULDB therefore accommodates greater payload mass by requiring relatively little ballast.

The ULDB gaseous envelope is a lightweight, thin film plastic material that has limited ability to accommodate stress. The pumpkin-shape design accommodates the meridional (vertical or longitudinal) stress component via external load tendons. The envelope is thus divided into gores that bulge outward with radius very small relative to the balloon radius (Figure 1), thereby greatly reducing the hoop (horizontal or latitudinal) stress in the film.

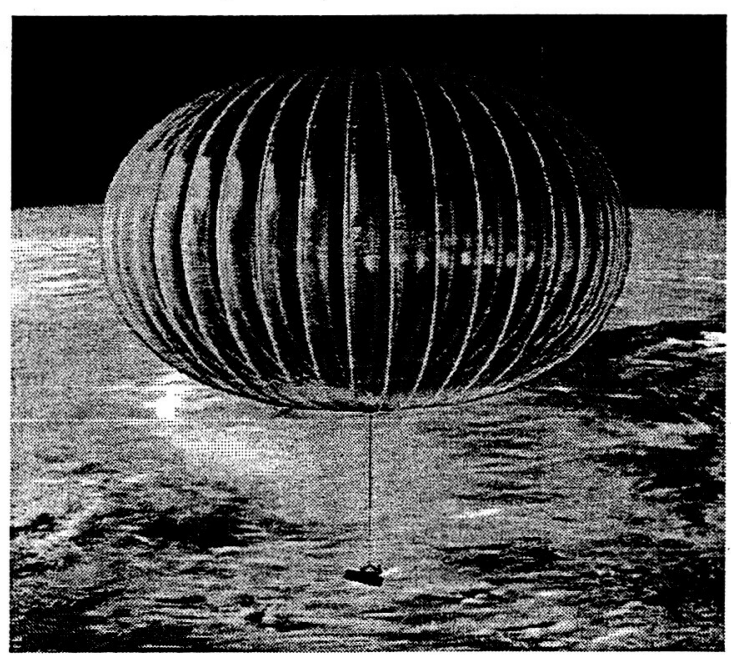

Figure 1. Artistic rendition of ULDB at float. 
The greatest advantage for the ULDB design can be met in part by the stiffest, strongest, lightest weight tendons that are resilient in the use environment. A liquid-crystalline polymer material, PBO fiber (tradename: Zylon ), was chosen for constructing the hollow-braided tendons, because it is the highest stiffness-to-weight and strength-to-weight material available, ${ }^{1,2}$ on the basis of information from the fiber manufacturer. ${ }^{3}$ Unfortunately, the available data on PBO primarily concems fibers or yarns, rather than the braided cord construction intended for ULDB. Industrial experience with PBO braided cord is extremely limited. Furthermore, there is little data available on PBO in any form that is independent of the manufacturer, and some of the available information suggests serious concerns over the resiliency of the material in the intended usage manner. ${ }^{3}$

Poly(para-phenylene-2,6-benzobisoxazole) (PBO) fiber is manufactured by Toyobo Co., Japan. The HM (high modulus) grade used for the ULDB tendons is derived from the AS (as spun) grade via heat treatment. The resulting molecules are rod-like and crystal orientation axially in the fiber is extremely high. These characteristics lead to the high tenacity (strength) and modulus (stiffness) of the fiber, and arise from the very stiff and planar chemical structure resulting from the high degree of aromaticity. ${ }^{4}$

If the fiber tensile strength reported by the manufacturer, $5.8 \mathrm{GPa}^{3}$ scaled directly, the braided cord used on ULDB should sustain in excess of 15 kilonewtons $(\mathrm{kN})$. Such scaling is widely known to be inappropriate, however, because reliable means of predicting cord strength from knowledge of the composing fiber strength do not exist, especially for a material as new as PBO. Nonetheless, a comparison of the theoretical cord strength ( $15 \mathrm{kN})$ to the maximum load measured during test flights $(\approx 4 \mathrm{kN})$ shows there may be significant opportunity for optimization. Although some loss of strength is expected for this material due to the braiding process and environmental degradation over time, the ratio ( $>3$ ) of utilized strength to available strength may be excessive.

NASA's Johnson Space Center (JSC) worked with $\mathrm{PBO}$ cord during two time frames. Omdoff studied it in the early 1990's in terms of its general potential for use by NASA, ${ }^{5}$ particularly in comparison to $\operatorname{Kevlar}^{*}$. Significant development of the material has occurred since that work, however the general information and qualitative behaviors discussed are confirmed by Toyobo's documentation. ${ }^{3}$ More recently, Maloy and Machin discussed the space rating of materials for the $\mathrm{X}-38$ project. ${ }^{6} \mathrm{PBO}$ cord and webbing, as well as other materials, were subjected to tensile analysis before and after exposure to light of various sources, radiation, and thermal vacuum cycling. Because their report did not give cord construction details such as braiding parameters, a direct comparison to the ULDB material is not possible.

There are numerous papers in the scientific literature discussing PBO fiber, ${ }^{7-9}$ and much of the information from these papers that is relevant to ULDB is covered in Toyobo's documentation. ${ }^{3}$ The literature does discuss the molecular deformation processes in much more depth, which helps understand the material even if it cannot yet be applied to vehicle design optimization.

The accurate characterization of the tendon material used in ULDBs is vital to the successful deployment and survival of the balloons. While well into the current ULDB design and testing phase, the present understanding of the PBO tendon strength is limited. Development of a sound mechanical properties measurement scheme will also facilitate analysis of the effects of material degradation. Additionally, a strategy must be developed to assess replacement materials well in advance of their potential integration into the ULDB construction. The purpose of this work was to develop a mechanical properties assessment protocol for ULDB tendon materials, and to assess PBO braided cord for this application.

\section{METHODS}

Organizations that provide material testing standards, such as the American Society for Testing and Materials (ASTM) and the International Standards Organization (ISO), were consulted to initiate protocol development. Standard testing procedures are typically intended as reference procedures for communication between suppliers and end-users, and thereby apply particularly well to quality control or quality assurance needs. This intention is somewhat applicable in the case of ULDB concerns. However, the over-riding principle is that the mechanical properties of polymeric materials vary with loading characteristics and therefore the measurement conditions should accurately mimic the application environment. ${ }^{10-12}$ Because there is little industrial experience with the subject material, the relevant testing standards remain a reasonable starting point for measurement methods and instrument specification.

ASTM is the marquee organization in the United States for standard analysis methods. ASTM D4268, "Standard Test Methods for Testing Fiber Ropes"10 initially appeared to be applicable, but it was cancelled in 2002 with no replacement or revision. Further investigation revealed that for this Standard, ASTM consulted The Cordage Institute, an industry consortium of fiber, rope, and rope-making equipment manufacturers. ASTM D4268 was closely tied to a Cordage Institute standard developed in 1993. The 
most recent version of this document, CI-1500-01," "Test Methods for Fiber Rope," is not specific to highmodulus fibers but provides guidelines that may be generally applicable.

The "Standard Test Methods for Tire Cords, Tire Cord Fabrics, and Industrial Filament Yarns Made from Manufactured Organic-Base Fibers," ASTM D885, ${ }^{12}$ provides advice specific to high-modulus synthetic materials. The title of the Standard suggests the target audience to which it is tailored. Nonetheless, there are portions of this Standard that apply to the PBO cord properties investigation.

The guidance from the testing and industry organizations mentioned above was compiled into the following general protocol.

- Measure properties at conditions as similar to the use environment as possible.

- Operate the load cell within 10\%-90\% of full scale.

- Operate the instrument in constant-extension-rate (CRE) mode.

- To determine breaking strength, set the crosshead speed such that $20 \%$ of the estimated breaking load is attained in $2-200$ seconds.

- Pretension rope at low load prior to further testing.

- For cordage under $16 \mathrm{~mm}$ in diameter, use at least 1' $300 \mathrm{~mm}$ gauge length.

- For spliced eyes, use pins of at least twice the nominal rope diameter.

- Discard specimens that fail within $3 \mathrm{~mm}$ of grips.

The protocol guidelines were then adapted into the general procedure.

- Carefully construct and identify the specimen.

- Inserted it into the instrument and remove the slack.

- From this unstressed state, apply load in a controlled manner until a specified load is attained.

- Hold this 'pretension load' for a specified time.

- Apply a controlled displacement rate to material failure.

The general procedure was further specified through a series of experiments to be discussed below.

\section{EXPERIMENT SETUP}

Various instruments were assessed for this project. The quality assurance test bed at the ULDB assembly contractor was not favored because its location would have implied either significant offsite time for NASA personnel or potential loss of scientific control. Other contractors were eliminated from consideration due to similar concerns. The option to purchase a custom instrument was not desirable due to high capital expenditure of approximately $\$ 100,000$.

The Balloon Research and Development Laboratory (BRDL) has a hydraulically-driven Instron ${ }^{2}$ tensile testing instrument (Model 8505) with software (Wavemaker ${ }^{8}$ ) for precise control and data acquisition. It is maintained according to ASTM guidelines. ${ }^{13,14}$ The instrument has an environmental chamber allowing experiments within $145-325 \mathrm{~K}$. It is oriented vertically and is thereby limited in maximum extension by issues of stability. Attempts to use standard tensile testing grips, such as the barrel design commonly used for ropes, were unsuccessful.

The ULDB tendons are affixed to the balloon apex and nadir plates using stainless steel pins which pass through eye splices tied in the cord. A Brummel splice, in which the cord passes through its own weave and the eye tail is buried within the hollow braid, is used. ULDB uses a 5" splice eye with minimum 18" tail bury to minimize splice failure rate. The PBO cord quality assurance (QA) testing protocol uses these splice parameters. The 10' QA specimen length was set by projecting that a gauge length at least three times the length of the splice would ensure cord breaking load, rather than splice effectiveness, would be assessed. To accommodate these long specimens, a custom horizontal test bed was constructed at the contractor's facility.

BRDL uses a smaller, but effective, splice to produce specimens within the size capability of its existing instrument. These splices have 40 " overall length, with 2" eye and 12" tail bury. Shorter eye length (1") resulted in failure in the splice, while shorter tail bury (10") resulted in splice slippage. Further verification that the smaller splice does not affect results is that the BRDL average for all tests is only slightly lower than the single test done at the contractor on the same spool of cord. The lower measurement, 11.5 versus $12.0 \mathrm{kN}$, is within the range of expected strength degradation over the time between the testing at the two facilities.

The specimens were connected to the instrument using a $3 / 8$ " diameter stainless steel pin to mimic the balloon construction. All measurements were done in laboratory ambient atmosphere (approximately $300 \mathrm{~K}$ and $50 \%$ relative humidity), except as noted for the exploration of low temperature properties. Due to the splicing, this setup measures extension of the entire specimen rather than gauge strain. Accurate measurement of the gauge strain would require a 2point measurement system, but the strain measurement is of secondary importance to the breaking load measurement. 


\section{RESULTS \& DISCUSSION}

The typical load-displacement curves in Figure 2 show that the material displays nearly linear behavior as expected. The curves show some offset, due to variation in the small amount of slack in the cord prior to pretensioning. The breaking load varies even among these specimens taken contiguously from the spool. The jaggedness in a few of the curves near the failure point indicates these specimens failed in stages, potentially due to uneven loading in the specimens. As some specimens neared failure, a noticeable 'ping' sound was heard, and it is suspected these sounds correlate with the jagged failures.

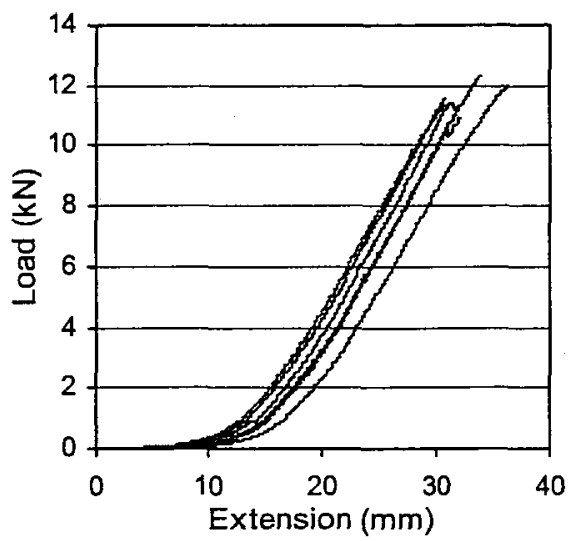

Figure 2. Typical load-extension curve for PBO cord showing expected near-linear behavior.

Typical loading profiles are shown in Figure 3, which addresses the investigation of varying the pretensioning parameters. As expected, instrument control was very good and the pretension load had no effect on the resulting breaking strength as shown in Table 1.

\begin{tabular}{|c|c|c|}
\hline $\begin{array}{c}\text { Pretension } \\
\text { Load, } \mathrm{N}\end{array}$ & \# specimens & $\begin{array}{c}\text { Break Load } \\
\mathrm{kN}\end{array}$ \\
\hline 10 & 6 & $11.4 \pm 0.5$ \\
\hline 100 & 10 & $11.7 \pm 0.5$ \\
\hline 1000 & 10 & $11.6 \pm 0.4$ \\
\hline
\end{tabular}

Table 1. Pretension load had no effect on the breaking strength.
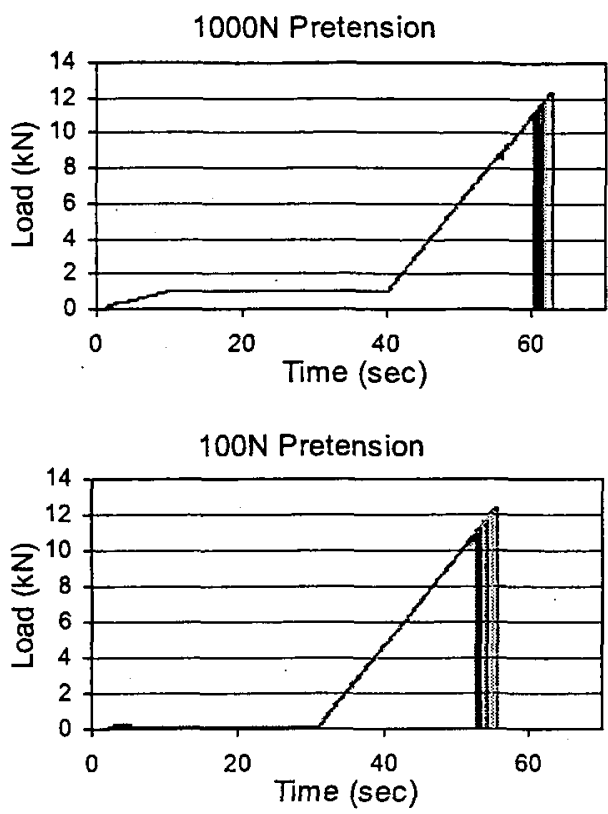

10N Pretension

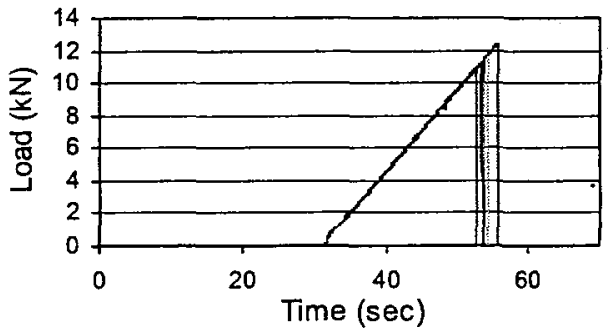

Figure 3. Loading profiles are consistent.

Polymer materials, particularly highly-oriented ones such as liquid crystalline fibers, are known to be strain-rate dependent. In this work, displacement or extension is used, rather than strain, because the instrument has no proper extensometer, so the extension includes the lengthening of both gauge and splice lengths. The key information to address the project requirements is failure load, as the material stiffness is very high, showing less than $3 \%$ strain at failure. Extension rates over the range $0.1-125 \mathrm{~mm} / \mathrm{min}$ were investigated for their effect on the failure load (Figure 4, Table 2). This range encompasses the rates suggested by the standards organizations, as well as those used in the QA procedures. There was statistically no measurable difference over the range $2.5-125 \mathrm{~mm} / \mathrm{min}$. The range was then extended to slower rates because the material is expected to display increased strength at higher rates, and the balloon flight dynamic is expected to be very slow. The slower rate showed appreciably lower failure loads. Because 
this slowest rate requires approximately 3 hours per experiment, no slower rates have been investigated.

\begin{tabular}{|c|c|c|}
\hline CRE mm/min & \# specimens & $\begin{array}{c}\text { Break Load } \\
\mathrm{kN}\end{array}$ \\
\hline 0.1 & 26 & $10.54 \pm 0.45$ \\
\hline 2.5 & 25 & $11.06 \pm 0.73$ \\
\hline 50 & 29 & $11.16 \pm 0.68$ \\
\hline 125 & 30 & $11.21 \pm 0.80$ \\
\hline Combined & 123 & $11.00 \pm 0.71$ \\
\hline
\end{tabular}

Table 2. Extension rate effect on break load. All experiments on Instron using "Spool 7" material.

Previously it was mentioned that a goal in designing experiments to assess polymeric materials is to closely mimic the use environment. There is little tendon load data from ULDB test flights, but they show that the slowest experiment rate mimics the fastest recorded balloon dynamic. In other words, to mimic flight conditions, slower experiments are needed. The limit of slower tensile experiments is tensile creep. Liquid crystalline fibers have a known property called creep rupture, in which the material fails under a creep load less than its ultimate load as determined from dynamic tensile measurements. The data on $\mathrm{PBO}$ from the manufacturer suggest this is a serious concern. ${ }^{3}$

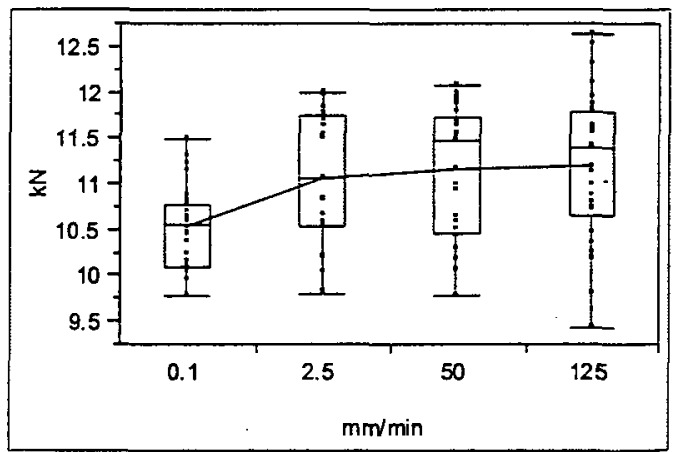

Figure 4. Displacement rate effect on failure load. Red: Box plots (ends of box are $25^{\text {th }}$ and $75^{\text {th }}$ quartiles, line across box middle is the median).

Blue: Means connected.

The vast majority of experiments discussed in this work were done in laboratory ambient conditions of approximately $23^{\circ} \mathrm{C}$. The ULDB at float is expected to be at -10 to $0^{\circ} \mathrm{C}$, but during ascent it passes through a region in which the temperature may be as low as -90 ${ }^{\circ} \mathrm{C}$. Therefore, the PBO cord breaking strength was investigated at this lowest expected temperature. The data (Table 3, Figure 5) show the cord is significantly stronger at the lower temperature, and that the effect of strain rate is somewhat muddled by the temperature effect.

\begin{tabular}{|c|c|c|c|}
\hline $\begin{array}{c}\text { CRE } \\
\mathrm{mm} / \mathrm{min}\end{array}$ & $\begin{array}{c}\text { Temperature } \\
{ }^{\circ} \mathrm{C}\end{array}$ & $\begin{array}{c}\# \\
\text { specimens }\end{array}$ & $\begin{array}{c}\text { Break } \\
\text { Load } \\
\mathrm{kN}\end{array}$ \\
\hline 0.1 & 23 & 26 & $10.5 \pm 0.4$ \\
\hline 1.0 & 23 & 13 & $11.0 \pm 0.6$ \\
\hline 0.1 & -90 & 22 & $13.6 \pm 1.0$ \\
\hline 1.0 & -90 & 23 & $13.9 \pm 0.8$ \\
\hline
\end{tabular}

Table 3. PBO cord is stronger at lower temperature.

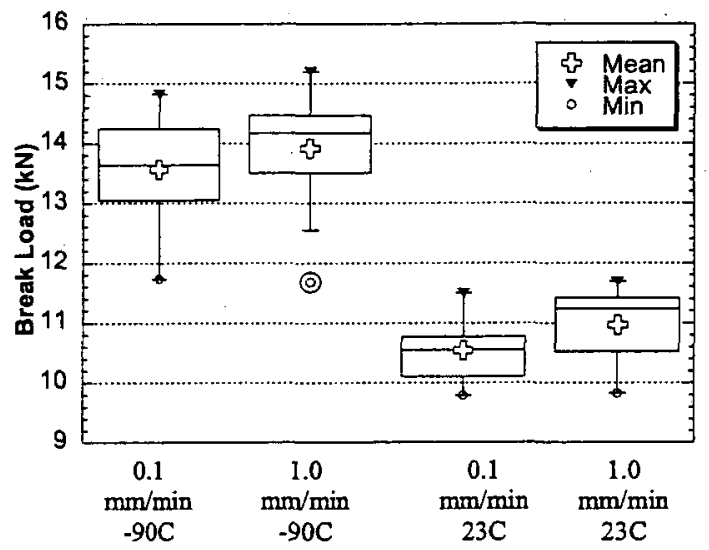

Figure 5. Effect of temperature on breaking strength of PBO cord. The double-circled data point is considered a statistical outlier.

To affirm any estimation of vehicle reliability, it is necessary to understand the native variation in the material. By carefully tracking the identification of each specimen relative to its origin in manufacturing, there is the possibility of showing strength variation within a spool of material. Figure 6 shows there is variability in the breaking load, but there appears to be no trend along the spool length that is separable from the effect of the differing strain rates. A statistical analysis suggested that many more specimens ( $>50)$ must be tested at the same extension rate to distinguish any spool-length variation. 


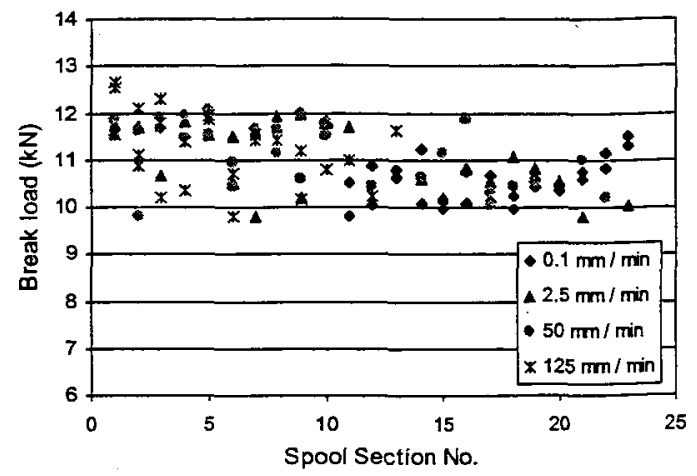

Figure 6. PBO cord strength data along an entire spool.

\section{CONCLUSION \& FUTURE WORK}

A protocol for studying PBO braided cord for application as the ULDB tendon material was developed based on general guidance from the literature combined with laboratory exploration. Experiments showed that the level and duration of pretensioning does not have significant effect on PBO cord breaking strength. The breaking strength is significantly higher at the lowest temperatures ULDB is expected to encounter than it is at

- laboratory conditions. The breaking strength is not sensitive to tensile strain rate within the range of rates suggested by the literature. However, when the strain rate is reduced to conditions similar to ULDB flight characteristics, the breaking strength decreases.

Future work may extend the investigation of slower tensile strain rates toward the limit of creep conditions, but may instead focus on the determination of creep lifetime under specified loading. Because ULDB tendons are much longer than the test specimens used to date, it seems wise to probe the effect of gauge length tensile strength. The effects and control of environmental ageing will also be investigated due to the well-known degradation of $\mathrm{PBO}$.

This work is closely related to an effort being led by Dr. Willi Schur of the New Mexico State University Physical Sciences Laboratory to develop a framework within which the results of this material characterization effort will be applied to ULDB design and operation. ${ }^{15}$ There will therefore be interaction between design modification and improved knowledge of material properties. This work may also lead to a reevaluation of the $\mathrm{PBO}$ cord quality assurance procedures.

\section{ACKNOWLEDGEMENTS}

The efforts of the Balloon Research \& Development Laboratory technicians, Lana Budd, Greg Parks, and Roy Tolbert, are greatly appreciated. The Balloon Program Office provided financial support and context for this work. Frequent communication with Dr. Willi Schur (New Mexico State University, Physical Sciences Laboratory), the principal ULDB designer, was particularly beneficial Tadao Kuroki (Toyobo Co.) provided information about PBO fiber. Dr. Canan Bilen-Green (North Dakota State University, NASA Summer Faculty Fellow), provided the statistical analyses.

\section{REFERENCES}

1. Yabuki, K., 12th Annual Meeting of the Polymer Processing Society (Sorrento), 1996.

2. Hokudah, T., K. Yabuki, T. Kuroki, and J. Nomura, 35th International Man-Made Fibers Conference (Dombirm), 1996.

3. Toyobo Co., Ltd., PBO Fiber Zylon Technical Information, 2001.

4. Kitigawa, T., H. Murase, and K. Yabuki, J. Polym. Sci. B: Polym. Phys., v.36, p.39-48, 1998.

5. Ondoff, E., NASA Technical Memorandum (TM104814), 1995.

6. Maloy, J., and R.A. Machin, ALAA Aerodynamic Decelerator Systems Technology Conference (Paper No. 2003-2174), Monterey, 2003.

7. Kitigawa, T., M. Ishitobi, and K. Yabuki, J. Polym. Sci. B: Polym. Phys., v.38, p.1605-1611, 2000.

8. Kitigawa, T., K. Yabuki, and R.J. Young, Polymer, v.42, p.2101-2112, 2001.

9. Davies, R.J., M.A. Montes-Moran, E. Reikel, and R.J. Young, J. Mat. Sci., v.36, p.3079-3087, 2001.

10. American Society for Testing and Materials, Standard Test Method for Fiber Ropes (ASTM D4268), cancelled 2002.

11. Cordage Institute, Test Methods for Fiber Rope (CI-1500-01), 2001.

12. American Society for Testing and Materials, Standard Test Methods for Tire Cords, Tire Cord Fabrics, and Industrial Filament Yarns Made from Manufactured Organic-Base Fibers (ASTM D885), 2002.

13. American Society for Testing and Materials, Standard Practices for Force Verification of Testing Machines (ASTM E4), 2002.

14. American Society for Testing and Materials, . Standard Specification for Tensile Testing Machines for Textiles (ASTM D76), 1999.

15. Schur, W.W., C. Bilen-Green, and W.J. Sterling, ALAA-ATIO (Paper No. 2003-6778), Denver, 2003 